\title{
Two-photon coherent control of atomic collisions by light with entangled polarization
}

\author{
M.D.Havey \\ Physics Department Old Dominion University \\ Norfolk, Virginia 23529
}

D.V.Kupriyanov and I.M.Sokolov

Department of Theoretical Physics, State Technical University, 195251, St.-Petersburg, Russia

\begin{abstract}
We describe a new method of coherent optical control of internal dynamics of atomic collisions by means of two correlated light beams having entangled polarizations. We show that if excitation of a colliding pair of atoms is by two photons having entangled polarizations, it is possible to redirect the output fragments of the collision into certain channels with a selected type of internal transition symmetry. The transition symmetry is defined in the body-fixed coordinate frame which has random and originally unknown orientation in space.

PACS numbers: $34.50 . \mathrm{Rk}, 34.80 . \mathrm{Qb}, 42.50 . \mathrm{Ct}$
\end{abstract}

Significant experimental developments over the past decade have led to remarkable understanding of details of atomic collision dynamics. Studies of a broad spectrum of processes, ranging from cold and ultracold collisions [1 3], photoassociation [4, 5], photodissociation [6, 7], photochemical reactions [8, 9], optical and fractional collisions [10, 11], and collisional redistribution of light 12 have revealed novel and often surprising effects depending on variables associated with the collision alone and with properties of the light used to initiate or probe the dynamics. In many cases, dynamical correlation of internal variables of the colliding particles have played a critical role in the outcome; for light-induced processes, dependencies on the cross sections due to classical characteristics of the light, viz. polarization, frequency and intensity have been determined. A novel method to obtain coherent control of the correlations in photodissociation, by using elliptically polarized light, demonstrated selectivity in the branching ratios for the process 7]. Further, general principles for coherent control of collision and reactive processes using a single light source have recently been developed in a fundamental paper on control of bimolecular scattering processes [13].

Due to recent advances in studies of photochemical processes on a femtosecond time scale [8, 9] and, as was recently shown in atomic collision experiments [10, 11], it becomes possible to optically probe a colliding system directly in the interaction domain and to select in this manner a small segment of a collision trajectory. Such a process, termed a fractional optical collision, is an example of a continuum-continuum two-photon spectroscopy where the first and the second photons are used for initiating and interrupting the collisional motion in an intermediate molecular state. In studies up to now, photoexcitation was driven by two independent light sources used mainly for selecting and probing the location of the Condon points of the fractional collision as described by quasistatic conditions of photoexcitation. The details of the internal collisional dynamics, as well as the selective information about different channels involved in the process were difficult to extract from the data obtained from the spectroscopic analysis, in spite of the fact that both intensity and polarization spectra were determined.

In the present letter, we describe how the outcome of a fractional collision may be significantly and selectively controlled by utilization of quantum-correlated light beams. Our approach is based on the requirement that the photon correlations must interfere with correlations arising from internal collisional dynamics. In our description of the light statistics we employ a fully quantal approach, which permits us to discuss the difference in predictions for classical and quantum electrodynamics. One aim of the present paper is to point out that to understand precise optical control of elementary processes like atomic collisions, it is important to follow the transformation of quantum correlations (existing on a wave function level) from an electromagnetic subsystem into a diatomic (or multi-atomic) subsystem. As a practical example of non-classical light, we consider the radiation from an optical parametric oscillator (OPO) operating in a subthreshold regime, and so having entangled polarizations of the output modes. Different schemes of practical realization of entangled states with the aid of optical parametric oscillators have been discussed in the literature [14 19].

In a perturbation theory approach any two-photon process can be described in terms of the light correlation function of second order, i.e. in terms of time- $(T)$ and anti-time- $(\tilde{T})$ ordered products of the Heisenberg operators of positive and negative frequency components of electric field amplitudes $E_{\nu}^{( \pm)}(\mathbf{r} t)$ considered as functions of space $(\mathbf{r})$ and time $(t)$ coordinates [20]. For a two-mode OPO output with entangled orthogonally polarized components, such a correlation function can be expanded in the following sum

$$
D_{\nu_{1} \nu_{2} ; \nu_{1}^{\prime} \nu_{2}^{\prime}}(\tau)=\left\langle\tilde{T}\left(E_{\nu_{1}^{\prime}}^{(-)}(\mathbf{r} t) E_{\nu_{2}^{\prime}}^{(-)}(\mathbf{r} t+\tau)\right) T\left(E_{\nu_{1}}^{(+)}(\mathbf{r} t) E_{\nu_{2}}^{(+)}(\mathbf{r} t+\tau)\right)\right\rangle=
$$




$$
\sum_{1,2,1^{\prime}, 2^{\prime}}\left(\mathbf{e}_{1}\right)_{\nu_{1}}\left(\mathbf{e}_{2}\right)_{\nu_{2}}\left(\mathbf{e}_{1}^{*}\right)_{\nu_{1}^{\prime}}\left(\mathbf{e}_{2}^{\prime *}\right)_{\nu_{2}^{\prime}} D_{121^{\prime} 2^{\prime}}(\tau)
$$

where each of the polarization vectors $\mathbf{e}_{i}, \mathbf{e}_{i}^{\prime}$ (with $i=1,2$ ) is one of the basic orthogonal polarizations of the OPO. The sum over $1,2,1^{\prime}, 2^{\prime}$ is restricted by the rule $\mathbf{e}_{1} \neq \mathbf{e}_{2}$ and $\mathbf{e}_{1}^{\prime} \neq \mathbf{e}_{2}^{\prime}$, so there are four terms in the expansion (11). We assume here steady state and homogeneous conditions of photoexcitation and consider the correlation function only as a function of the time delay between appearances of the first and second photons.

Strictly speaking the above expansion of the full correlation function relates to the limit of weak sub-threshold OPO source, generating the photon pairs, see Eq.(7). This is the most interesting and important case for our discussion. But in a more general situation, to introduce the expansion (11), we need to cancel out the non-correlated contribution when both the photons appear in the same polarization mode. However even in a general situation, for methodical clarity, it is useful to discuss the correlation function in form (1) since it lets us compare the difference between quantum and classical types of polarization entanglement.

A schematic diagram illustrating the process of two-photon excitation of colliding atoms is shown in Figure 1. There the vertical lines represent optical transitions, while the paths along the interatomic potentials indicate the kinetic motion of the colliding atoms. Based on the Franck-Condon approximation and on the assumption of adiabatic evolution of the diatomic system in the intermediate states, the total cross-section (or transition probability) of the fractional collision can be expressed as follows

$$
\begin{gathered}
\sigma_{0}=\sum_{1,2,1^{\prime}, 2^{\prime}} \sum_{X \Xi}(-)^{X+\Xi} \Phi_{X \Xi}\left(\mathbf{e}_{1}, \mathbf{e}_{1}^{*}\right) \Phi_{X-\Xi}\left(\mathbf{e}_{2}, \mathbf{e}_{2}^{*}\right) \times \\
Q_{121^{\prime} 2^{\prime}}^{(X)}
\end{gathered}
$$

where the tensor functions

$$
\begin{aligned}
\Phi_{X \Xi}\left(\mathbf{e}, \mathbf{e}^{\prime *}\right)= & -\sum_{\nu, \nu^{\prime}} \mathrm{C}_{1 \nu^{\prime} 1 \nu}^{X \Xi}\left(\mathbf{e}^{\prime *}\right)_{\nu^{\prime}} e_{\nu}= \\
& \sum_{\nu, \nu^{\prime}}(-)^{1+\nu^{\prime}} \mathrm{C}_{1 \nu^{\prime} 1 \nu}^{X \Xi} e_{-\nu^{\prime}}^{\prime *} e_{\nu},
\end{aligned}
$$

considered as a function of $\mathbf{e}, \mathbf{e}^{\prime}=$ either $\mathbf{e}_{1}, \mathbf{e}_{1}^{\prime}$ or $\mathbf{e}_{2}, \mathbf{e}_{2}^{\prime}$, are the irreducible polarization components of the OPO light. Here by $\mathrm{C}_{\ldots \ldots} \ldots$ we denote the Clebsch-Gordan coefficients in the notation of Ref. [21]. Each partial contribution of the $X$-rank components in the irreducible product in Eq.(2) is weighted with the factor $Q_{121^{\prime} 2^{\prime}}^{(X)}$ given by

$$
\begin{aligned}
& Q_{121^{\prime} 2^{\prime}}^{(X)} \sim \frac{1}{\left(2 j_{0}+1\right)(2 X+1)} \sum_{\bar{\Xi}} \sum_{\bar{\nu}_{1} \bar{\nu}_{1}^{\prime}} \sum_{\bar{\nu}_{2} \bar{\nu}_{2}^{\prime}}(-)^{\bar{\nu}_{2}^{\prime}+\bar{\nu}_{1}} \mathrm{C}_{1 \bar{\nu}_{2} 1-\bar{\nu}_{2}^{\prime}}^{X \overline{\bar{S}}} \mathrm{C}_{1-\bar{\nu}_{1} 1 \bar{\nu}_{1}^{\prime}}^{X \overline{\bar{g}}} \times \\
& w^{(1)} w^{(2)}\left[\mathrm{d}_{\bar{\Xi} \bar{\Xi}}^{X}\left(\xi_{+-}\right) \mathcal{D}_{121^{\prime} 2^{\prime}}\left(\tau_{+-}\right)+\mathrm{d}_{\bar{\Xi} \bar{\Xi}}^{X}\left(\xi_{-}\right) \mathcal{D}_{121^{\prime} 2^{\prime}}\left(\tau_{-}\right) \theta\left(R_{1}-R_{2}\right)+\right. \\
&\left.\mathrm{d}_{\bar{\Xi} \bar{\Xi}}^{X}\left(\xi_{+}\right) \mathcal{D}_{121^{\prime} 2^{\prime}}\left(\tau_{+}\right) \theta\left(R_{2}-R_{1}\right)\right]
\end{aligned}
$$

where

$$
\mathcal{D}_{121^{\prime} 2^{\prime}}(\tau)=(\operatorname{Tr} D(\infty))^{-1} D_{121^{\prime} 2^{\prime}}(\tau)
$$

is the dimensionless correlation function normalized according to its classical limit. With reference to Fig. 2., the following notation is used in Eq.(44). First, $j_{0}$ is the angular momentum of the lower state. In the arguments of the Wigner $d-$ function $\xi_{+-}$and $\xi_{ \pm}$are the average deflection angles defined for different segments of the collisional trajectory crossing the Condon points $R_{1}$ and $R_{2}$, as defined in Fig. 2. $\tau_{+-}$and $\tau_{ \pm}$are the average durations of the fractional collision defined for these segments of the trajectory, while $w^{(1)}$ and $w^{(2)}$ are the Franck-Condon transition probabilities for optical excitation near the points $R_{1}$ and $R_{2}$ respectively. The step $\theta$-functions in Eq.(4) indicate that such transitions are acceptable either on incoming or outgoing parts of the motion.

The partial cross section $Q_{121^{\prime} 2^{\prime}}^{(X)}$ is the most important characteristic of the fractional collision process. As follows from Eqs.(1), (2), this quantity describes both the total probability for and the polarization dependence of the process. The expression (44) can be consistently derived based in the general theory of fractional optical collisions [22]. Here we present only a qualitative description. First, we point out that all the tensor components in the sum of expression (41) relate to the internal molecular (body-fixed) frame; the corresponding tensor indices are indicated by over bars. 
The Clebsch-Gordan coefficients in this expression can be treated as irreducible components of the light as defined in the molecular frame. Second, the sum over tensor indices is non-invariant here and is expanded only over those transitions which are permitted by the Franck-Condon principle. The Wigner $d$-functions describe the rotational and adiabatic transformation of the light irreducible components due to adiabatic evolution of the electronic subsystem during the internal part of the collision for atomic motion from point $R_{1}$ to point $R_{2}$.

The correlation function of the light appears in Eq. 4 as a function of fractional collision time intervals, which reveals how the photon correlations interfere with the collisional dynamics. Consider the situation when the photons, emitted by a subthreshold OPO, can appear in two orthogonal polarizations along $x$ and $y$ axes: $\mathbf{e}_{x}$ and $\mathbf{e}_{y}$. Then, assuming quasi-Gaussian statistics in the averaged product (1), we obtain the following set of dimensionless correlation functions:

$$
\begin{aligned}
& \mathcal{D}_{x y x y}(\tau)=\mathcal{D}_{y x y x}(\tau)=\frac{1}{2}+\frac{1}{2} \operatorname{coth}^{2} \kappa g(\tau) \\
& \mathcal{D}_{x y y x}(\tau)=\mathcal{D}_{y x x y}^{*}(\tau)=\frac{1}{2} \mathrm{e}^{-i \varphi} \operatorname{coth}^{2} \kappa g(\tau)
\end{aligned}
$$

where $\varphi$ is a phase mismatch between the anomalous correlation functions in the product

$$
\left\langle\tilde{T} E_{1 y}^{(-)}(\mathbf{r} t) E_{2 x}^{(-)}(\mathbf{r} t+\tau)\right\rangle\left\langle T E_{2 y}^{(+)}(\mathbf{r} t+\tau) E_{1 x}^{(+)}(\mathbf{r} t)\right\rangle
$$

Here in the indices we display both the mode number and the polarization. In our simple model of a subthreshold OPO, $\sinh ^{2} \kappa$ is of order of the number of photons emitted by the crystal in the coherence volume of parametric radiation. The opposite limits, when the efficiency of the process $\kappa \rightarrow 0$, or when $\kappa>1$, describe the weak and strong output respectively. Time correlation of twin-photons is described by the function $g(\tau)$ which can be controlled with an optical delay line.

From the point of view of classical electrodynamics only the first term (i.e. $\frac{1}{2}$ ) in the expression for the diagonal components of the correlation functions (6) is acceptable. The non-classical behavior of the correlation functions (6) can be clearly seen in the weak radiation limit: if $\kappa \rightarrow 0$ then $\operatorname{coth}^{2} \kappa \rightarrow \infty$. Actually, such a singularity means that for weak OPO light the correlation function (1) has linear (not quadratic) dependence on mode intensity. In this case the dimensionless correlation function, normalized in accordance to (5), should approach infinity for short time delay. The linear dependence of two-photon absorption on light intensity for the radiation created in the down-conversion process was recently observed in experiment [23]. For our discussion it is more important to point out the non-classical behavior of the polarization for OPO light. Indeed, in the limit of weak output the two-mode light illuminating the colliding atoms describes the photon pairs with the following cooperative wave function:

$$
|\Psi\rangle_{12}=\frac{1}{\sqrt{2}}\left[\left|\mathbf{e}_{x}\right\rangle_{1}\left|\mathbf{e}_{y}\right\rangle_{2}+\mathrm{e}^{i \varphi}\left|\mathbf{e}_{y}\right\rangle_{1}\left|\mathbf{e}_{x}\right\rangle_{2}\right]
$$

For such a polarization-entangled wave function in the case of $\varphi=\pi$ there is no particular polarization for each photon, but there is a strong mutual orthogonal polarization between them.

As a particular application, consider the collisional system, often discussed in optical collision theory, for which the optical transitions are initiated between singlet states of one atom. The second atom (an inert-gas atom) conserves its electronic configuration during the collision. If the optically active atom is originally in the ground ${ }^{1} S$-state there are the following dipole-allowed two-photon transitions available: ${ }^{1} S \rightarrow{ }^{1} P \rightarrow{ }^{1} S,{ }^{1} D$. For simplicity, let us ignore rotational effects and assume that the main contribution to the transition probability comes from the recoil collision with small impact parameters. Then we can substitute expressions (6) into Eqs.(4) and (2) and look at the partial contribution for each possible pair of Franck-Condon transitions. In the Franck-Condon approximation we can select all the acceptable transitions in terms of their molecular symmetry. Also in the recoil limit we can ignore the difference between incoming-outgoing and either incoming or outgoing parts of the classical trajectory, since all of them have similar polarization dependence.

With such assumptions the partial contribution to the cross-section for the excitation via ${ }^{1} \Sigma \rightarrow{ }^{1} \Sigma \rightarrow{ }^{1} \Sigma$ or ${ }^{1} \Sigma \rightarrow{ }^{1} \Pi \rightarrow{ }^{1} \Sigma$ is given by

$$
\sigma_{0} \propto \frac{1}{15} w^{(1)} w^{(2)}\left[1+(1+\cos \varphi) \operatorname{coth}^{2} \kappa g(\tau)\right]
$$

For the excitation via ${ }^{1} \Sigma \rightarrow{ }^{1} \Sigma \rightarrow{ }^{1} \Pi$ or ${ }^{1} \Sigma \rightarrow{ }^{1} \Pi \rightarrow{ }^{1} \Pi$ we obtain

$$
\sigma_{0} \propto \frac{4}{15} w^{(1)} w^{(2)}\left[1+\left(1-\frac{1}{4} \cos \varphi\right) \operatorname{coth}^{2} \kappa g(\tau)\right]
$$


and the contribution for the excitation via ${ }^{1} \Sigma \rightarrow{ }^{1} \Pi \rightarrow{ }^{1} \Delta$ channel is given by

$$
\sigma_{0} \propto \frac{1}{5} w^{(1)} w^{(2)}\left[1+(1+\cos \varphi) \operatorname{coth}^{2} \kappa g(\tau)\right]
$$

The dependence of these expressions on phase $\varphi$ reveals how the quantum correlations, existing between the OPO mode polarizations, can interfere with the internal dynamics of the fractional collision process. This is most easily seen in the limit of weak radiation, when the second terms in the brackets of Eqs.(8)-(10) give dominant contributions. If the phase $\varphi=\pi$, the transition probability for ${ }^{1} \Sigma \rightarrow^{1} \Sigma \rightarrow{ }^{1} \Sigma,{ }^{1} \Sigma \rightarrow{ }^{1} \Pi \rightarrow{ }^{1} \Sigma$ and ${ }^{1} \Sigma \rightarrow{ }^{1} \Pi \rightarrow{ }^{1} \Delta$ excitation channels drops sharply. Such behavior can be understood, based on the wave function (17), where the entangled states have unknown polarization for each photon, but there is a strong mutual correlation between their polarizations. If $\varphi=\pi$ and the first photon possesses unknown polarization along an arbitrary direction in the space then the second photon has polarization orthogonal to this direction. In such a case, the absorption of the first photon during the collision fixes the polarization direction for the second photon, i.e. reduces the uncertainty of its quantum state. Thus, when $\varphi=\pi$, the second photon has orthogonal polarization to the direction of the transition dipole moment. Then it cannot be absorbed in the above examples of the Franck-Condon transitions and such excitation channels become closed. But at the same time, the excitations via ${ }^{1} \Sigma \rightarrow{ }^{1} \Sigma \rightarrow{ }^{1} \Pi$ and ${ }^{1} \Sigma \rightarrow{ }^{1} \Pi \rightarrow{ }^{1} \Pi$ channels are open and we obtain in contrast an increase in the transition probability. Let us emphasize here that, from the point of view of classical electrodynamics, this effect is forbidden, since it would be impossible to prepare light polarized along (or orthogonal to) an arbitrary and originally unknown direction in space.

In summary, we have shown that unique manipulations of colliding atoms by light with entangled polarization can result in coherent control of elementary processes such as atomic collisions or chemical reactions. Even after full averaging the polarization entanglement makes it possible to close one channel of a photochemical reaction and to open another if they have different optical transition symmetries.

Support of this research by the U.S. Civilian Research and Development Foundation, the National Science Foundation and the Russian Foundation for Basic Research is gratefully acknowledged. D.K. would like to acknowledge the financial support from Delzell Foundation, Inc.

[1] J. Weiner, V.S. Bagnato, S. Zilio, and P.S. Julienne, Rev. Mod. Phys. 71, 1 (1999).

[2] K. Burnett, P. Julienne, P. Lett, K-A. Suominen, Phys. World 8, 42 (1995).

[3] T. Walker and P. Feng, Adv. At. Mol. Opt. Phys. 34, 125 (1994).

[4] J.J. Blange, J.M. Zijlstra, A. Amelink, X. Urbain, H. Rudolph, P. van der Straten, H.C.W. Beijerinck, and H.G.M. Heideman, Phys. Rev. Lett. 78, 3089 (1997).

[5] K.M. Jones, S. Maleki, L.P. Ratliff, and P.D. Lett, J. Phys. B: At. Mol. Opt. Phys. 30, 289 (1997).

[6] V. Zafiropulos, P.D. Kleiber, K.M. Sando, X. Zeng, A.M. Lyrra, and W.C. Stwalley, Phys. Rev. Lett. 61, 1485 (1988).

[7] C. Asaro, P. Brumer, M. Shapiro, Phys. Rev. Lett. 60, 1634 (1988).

[8] M. Dantus, M.J. Rosker and A.H. Zewail, J. Chem. Phys. 87, 2395, (1987); A.H.Zewail, J.Phys.Chem. 97, 12427 (1993).

[9] "Femtochemistry 97", Special issue of Phys. Chemistry A 102, \#23 (1998).

[10] D.A. Olsgaard, M.D. Havey, A. Sieradzan, R.A. Lasell, Phys. Rev. Lett. 69, 1745, (1992); Phys.Rev. A 48, 1987 (1993).

[11] M.D. Havey, S.B. Bayram, D.A. Olsgaard, H.O. Thurman, D.V. Kupriyanov, I.M. Sokolov, A.V. Slavgorodskii, A.I. Trubilko, Optics \& Spectr. 87, 683 (1999).

[12] K. Burnett, Phys. Rep. 118, 339 (1985).

[13] M. Shapiro, P. Brumer, Phys. Rev. Lett. 77, 2574 (1996).

[14] M.D. Reid, P.D. Drummond, Phys. Rev. Lett. 60, 2731 (1988).

[15] Z.Y. Ou, S.F. Pereira, H.J. Kimble, K.C. Peng, Phys. Rev. Lett. 68, 3663, (1992).

[16] B. Yurke, D. Stoler, Phys. Rev. A 46, 2229 (1992); Phys. Rev. Lett. 68, 1251 (1992).

[17] M. Zukowski, A. Zeilinger, M.A. Horne, A.K. Ekert, Phys. Rev. Lett. 71, 4287 (1993).

[18] Y.H. Shih, A.V. Sergienko, M.H. Rubin, T.E. Kiess, C.O. Alley, Phys. Rev. A 50, 23 (1994)

[19] P.G. Kwiat, K. Mattle, H. Weinfurter, Phys. Rev. Lett. 75, 4337 (1995); P.G. Kwiat et al., Phys. Rev. A 60, R773 (1999).

[20] R. Glauber, Optical Coherence and Photon Statistics in Quantum Optics and Electronics (New York: Gordon and Breach, 1965).

[21] D.A. Varshalovich, A.N. Moskalev and V.K. Khersonskii, Quantum Theory of Angular Momentum (World Scientific, Singapore, 1988).

[22] D.V. Kupriyanov, I.M. Sokolov, A.I. Trubilko, A.V. Slavgorodskii, Phys. Rev. A 60, 2230 (1999).

[23] N.Ph. Georgiades, E.S. Polzik, K. Edamatsu, H.J. Kimble, A.S. Parkins, Phys. Rev. Lett. 75, 3426 (1995). 


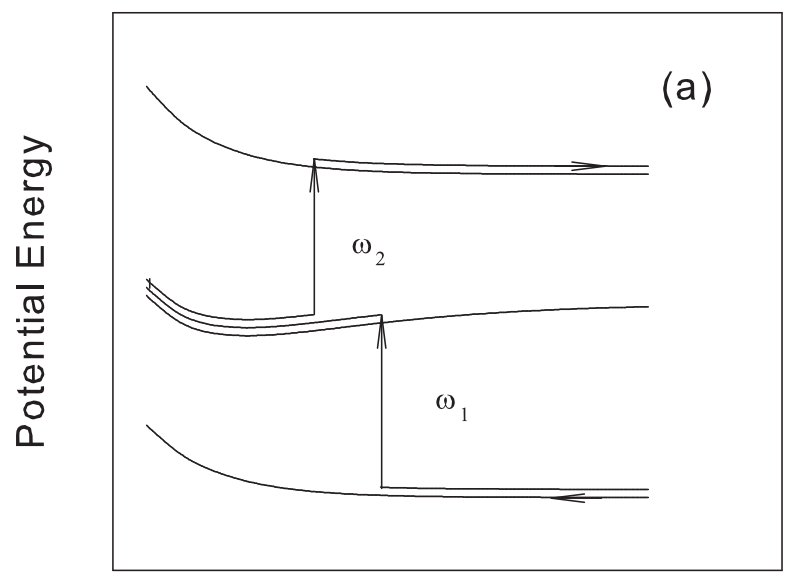

$\mathrm{R}$

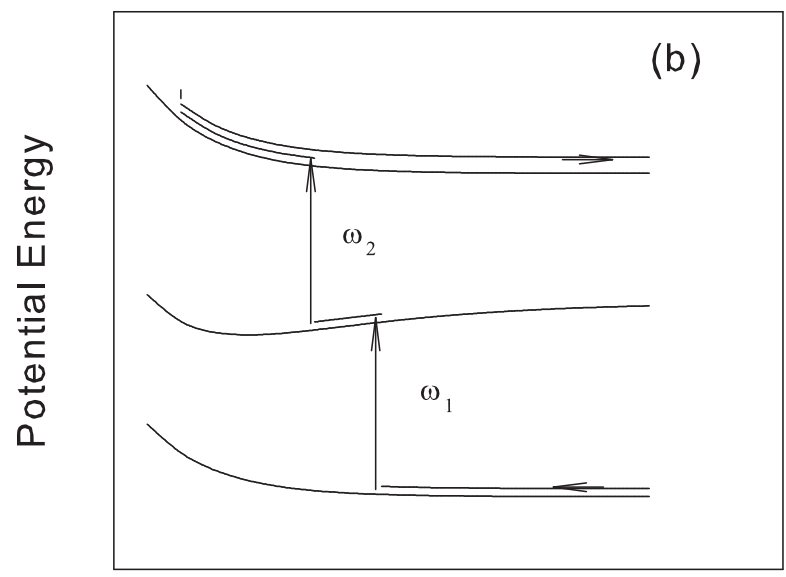

$\mathrm{R}$

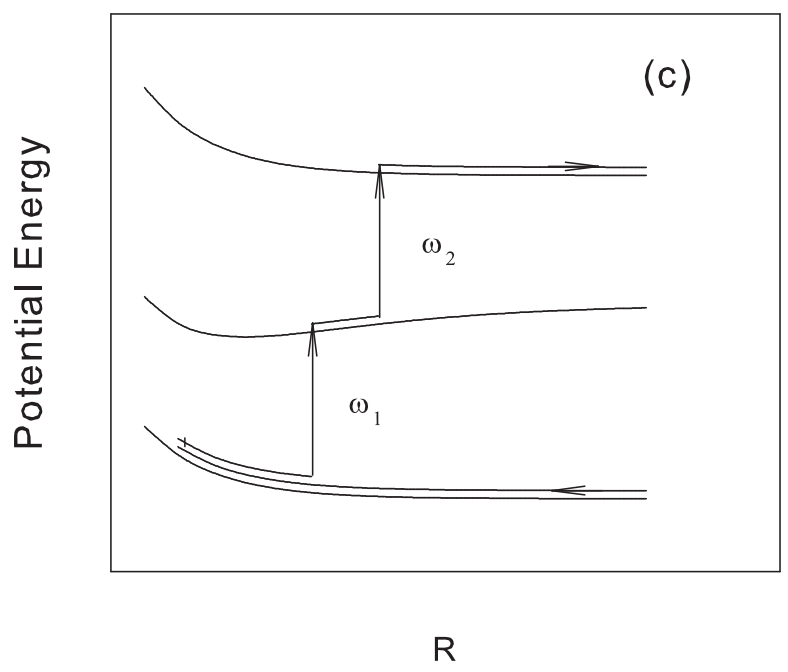

FIG. 1: Two-photon excitation of colliding atoms for locations of the Condon points on (a) incoming-outgoing, (b) incoming and (c) outgoing parts of a classical trajectory. 


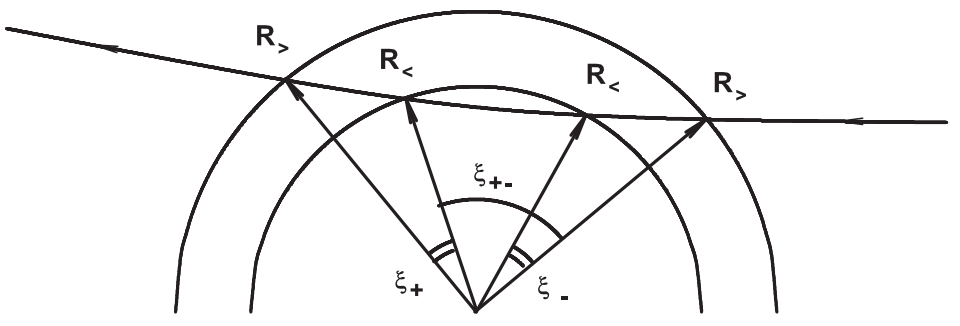

FIG. 2: Definition of deflection angles for different segments of a collisional trajectory. Here $R_{>}$or $R_{<}$is either $R_{1}$ or $R_{2}$ depending on the situations shown in Fig.1. 